\title{
ANOMALÍAS DE LA PRECIPITACIÓN PLUVIAL DE SÃO PAULO
}

\author{
Jonas Teixeira Nery \\ Campus Experimental de Ourinhos \\ Universal Estadual Paulista (UNESP, Brasil)
}

\section{RESUMEN}

El objetivo de este trabajo fue analizar la precipitación pluvial del Estado de São Paulo, basado en datos obtenidos junto a la Agencia Nacional de Agua (ANA). El período de análisis fue desde 1974 a 2003.

Basado en estos cálculos se puede concluir que las lluvias en el Estado sufren variabilidad espacial, temporal y que la explicación de esta variabilidad espacial está, muchas veces, asociada a la ocurrencia de eventos como El Niño y La Niña.

Palabras claves: variabilidad, anomalías, precipitación, ENOS.

\section{ABSTRACT}

The purpose of this paper is to analyses a rainfall in S. Paulo State. Monthly rainfall data during the period from 1974 to 2003. Rainfall during periods of El Niño and La Niña occurrence were compared with periods when these phenomena did not occur.

The result showed that a rainfall in S. Paulo State suffered special and temporal variability. The warm event of ENSO (El Niño) is favorable more precipitation in this area.

Key words: variability, anomaly, rainfall, ENSO.

\section{Introducción}

Brasil, por ser un país de gran extensión territorial, posee diferentes regímenes de precipitación y temperatura. De Norte a Sur se encuentran gran variedades de climas con distintas características regionales. En el Norte del país se observa un clima ecuatorial lluvioso, prácticamente sin estación seca. En la región Noreste la estación lluviosa tiene bajos índices pluviométricos se restringe a pocos meses, caracterizando un clima semiárido. Las regiones Sureste y Centro este sufren influencias tanto de sistemas tropicales como de 
latitudes medias, con estación seca bien definida en invierno y estación lluviosa en verano con lluvias convectivas. El Sur de Brasil, sufre, principalmente influencia de los sistemas de latitudes media, donde los sistemas frontales son los principales causadores de lluvias durante el año.

El Sureste, debido a su localización latitudinal se caracteriza por ser una región de transición entre climas cálidos de latitudes bajas y climas mesotermicos de tipo templado en latitudes medias (Nimer, 1979). El Sur de la región Sureste es afectada por la mayoría de los sistemas sinópticos que atingen el sur del país, con algunas diferencias entre los términos de intensidad y estacionalidad del sistema. Según Fernandes y Satyamurty (1994), las vaguadas invertidos actúan básicamente durante el invierno provocando condiciones de tiempo moderado, principalmente sobre el Mato Grosso del Sur y São Paulo. Vértice ciclónico en altos niveles oriundos de la región del Pacífico, se organizan con intensa convección asociadas a la inestabilidad causada por el Jato Subtropical. Línea de inestabilidad prefrontal, generadas a partir de la asociación de factores dinámicos de gran escala y características de mesoescala, son responsables por intensas precipitaciones (Cavalcanti et al., 1982).

La región Sudeste está caracterizada por la actuación de sistemas que asocian características de sistemas tropicales con sistemas típicos de latitudes medias. Durante los meses de mayor actividad conectiva, la Zona de Convergencia del Atlántico Sur (ZCAS) es uno de los principales fenómenos que influyen en el régimen de lluvias de esas regiones (Quadro y Abreu, 1994). El hecho de las bandas de nebulosidad y lluvias permanecieren semi - estacionarias por días seguidos favorece la ocurrencia de inundaciones en las áreas afectadas.

Los índices pluviométricos exhibidos por el Estado de São Paulo varían, desde cuotas excepcionalmente altas como aquellas presentadas por las lomas de la Sierra del Mar (2000 a $4500 \mathrm{~mm}$, anuales) hasta índices moderados como aquellos que son conocidos en ciertos trechos de la depresión periférica (de 1000 a 1100 mm anuales).

El trabajo realizado por Alves (2005), presenta patrones atmosféricos y oceánicos dominantes en el inicio de la estación lluviosa de la región Sudeste de Brasil, bien como su variabilidad interanual, a partir del análisis conjunta de los datos de precipitación producidos por el Climate Prediction Center de la NOAA.

Minuzzi (2004) estudió datos diarios de 134 localidades de Minas Gerais, con el objetivo de caracterizar el inicio del período lluvioso, la cantidad de lluvias durante el período lluvioso y el comportamiento de la ocurrencia y duración de los veranicos en años de ocurrencia de los fenómenos La Niña. Ya Catalunha (2002), analizó la distribución de probabilidad exponencial, gama, log-normal, normal y Weibull en series de precipitación pluvial en el Estado de Minas Gerais.

El objetivo de este trabajo es hacer un análisis de la variabilidad de la precipitación pluvial del Estado de São Paulo, en los últimos 30 años. Analizar las anomalías de la precipitación (variabilidad temporal) y la variabilidad espacial de la lluvia en el referido estado.

\section{Metodología}

Fueron analizadas series pluviométricas del Estado de Sao Paulo. Esos datos fueron obtenidos a través del sitio de Agencia Nacional de Água (ANA), utilizando el HIDROWEB, para seleccionar las informaciones. El período analizado fue de 1974 a 2003, buscándose la mejor distribución espacial (Figura 1). 


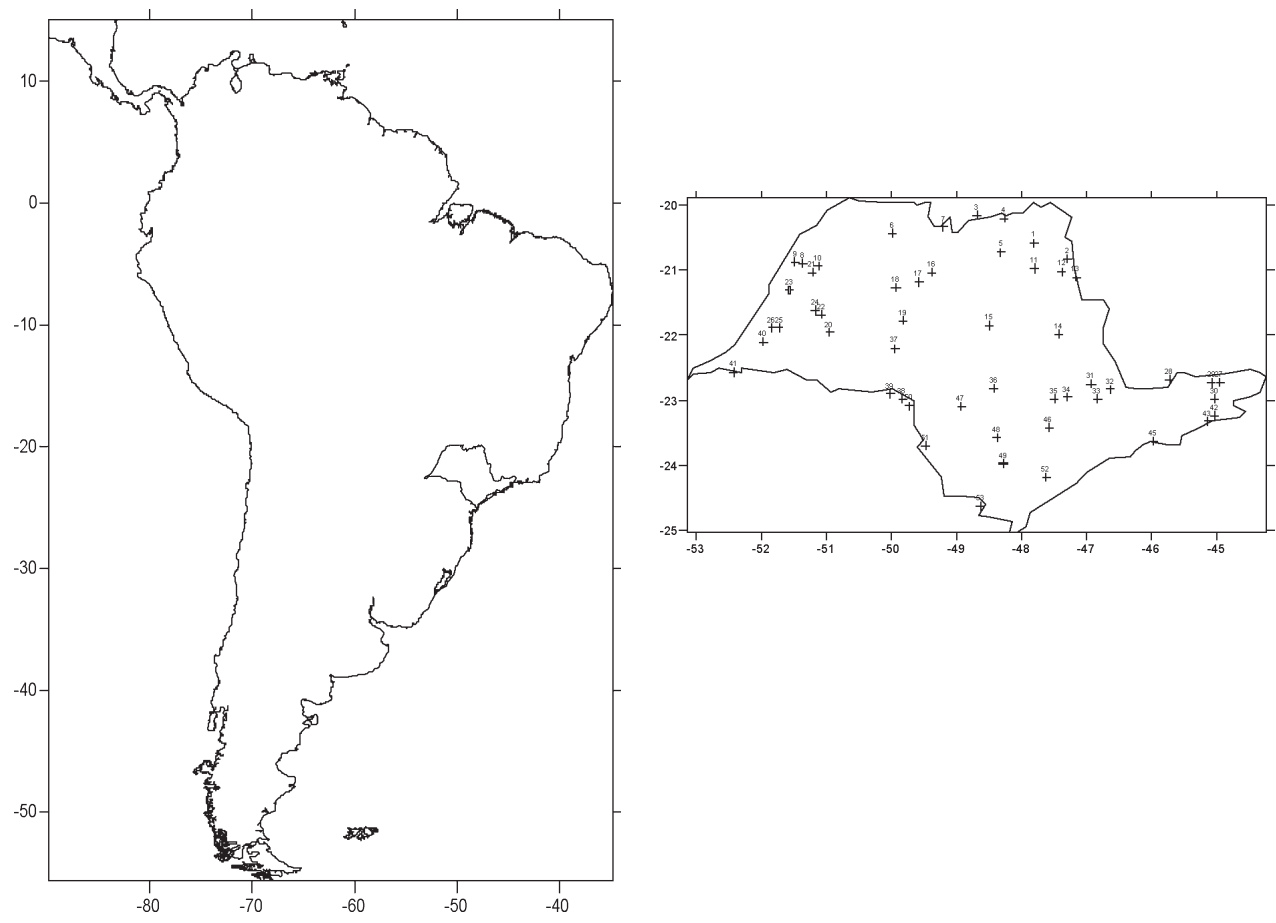

Figura 1. Distribución espacial, de las series pluviométricas analizadas en este trabajo.

Primero se calcularon los valores medios y desviaciones estándar dentro del período analizado, a continuación se hizo el cálculo de anomalías y se trazaron las isolíneas de estas anomalías para diferentes años.

También fue calculado un índice denominado de Índice Pluvial (IPP):

$$
I P P=\frac{P_{i}-\bar{P}}{\bar{P}}
$$

Siendo el valor de cada precipitación pluvial ocurrida en el mes o en el año, de acuerdo com el cálculo que se quiera realizar; $P$ es la precipitación pluvial del período analizado.

A seguir, utilizándose del análisis multivariado se obtuvieron áreas homogéneas, o sea, áreas que representaban el mismo régimen de precipitación pluvial.

El Análisis Multivariado es una herramienta estadística que procesa las informaciones de una forma que simplifica la estructura de los datos y sintetiza las informaciones cuando el numero de variables consideradas es muy grande, facilitando el entendimiento del racionamiento existente entre las variables del proceso.

Las regiones homogénea de precipitación pluvial fueron determinadas a partir de un Análisis de Componentes Principales (ACP), modo S (Richman, 1986), seguida de un análisis de un Análisis de Cluster (AC).

Las aplicaciones de la ACP, a un conjunto de datos com un gran números de variables, es interesante para determinar combinaciones lineales de variables originales, que explican 
lo máximo posible la variación existente en los datos iniciales (Reis, 1997). Com la ACP se elimina parte de la dificultad para interpretar los resultados obtenidos, pues la ACP elimina las informaciones redundantes y separa la señal de gran escala (variación común) del ruido (variación especifica) asociadas a cada estación y proporciona un análisis exenta de subjetividad (Silva y Nery, 2000).

La estrategia de proceder AC presenta dos grandes ventajas: a) a AC es más específicamente adaptada para construir agrupamientos (dendogramas); b) limita la parte de subjetividad en la elección de lo agrupamientos sugeridos por los planos factoriales. Hay varios criterios para determinar el numero de componentes principales $(\mathrm{CP})$ a retener.

\section{Análisis de los resultados}

En las Figuras 2 y 3, son presentados las isolíneas de los valores medios y del desviaciones estándar, para las series pluviométricas analizadas, dentro del periodo estudiado. Se puede observar que las precipitaciones medias son mayores al sudeste del Estado, disminuyendo al interior, as Figuras 2.

Los mayores valores medios están en la costa, con valores superiores a $1.800 \mathrm{~mm}$ y los menores valores están al noroeste, com valores inferiores a $1.300 \mathrm{~mm}$. La mayor variabilidad está también en la costa, com valores superiores a $320 \mathrm{~mm}$, al Este del Estado y los valores menores al $230 \mathrm{~mm}$.

En las Figuras 4 y 5 son trazadas isolineas del coeficiente de variación (Figura 4) y de las amplitudes intercuartiles (Figura 5), dentro del período del estudio. Se pueden e observar las isolineas del coeficiente de variación que el máximo distanciamiento del desvío en relación a la media climatológica (el coeficiente explica cuanto el valor calculado se aleja de la media, o sea, mide la variabilidad de la muestra analizada). Se observa que los valores del coeficiente oscilaron entre 16 e $24 \%$, siendo los menores valores al Este del Estado y los mayores valores (24\%), al Sudeste del área analizada. Figura 5, son presentados los valores de la amplitud intercuartil, observándose amplitudes de $440 \mathrm{~mm}$ (región Sudeste) a $220 \mathrm{~mm}$, en la región Sudoeste, de esta área analizada. Esos valores de amplitud analizan la variabilidad espacial para valores de precipitación pluvial, entre $25 \%$ (superior a $25 \%$ de lluvia) y $75 \%$ (valores inferiores a $75 \%$ de lluvia), o sea, aptitudes dentro del intervalo retirando los $25 \%$ extremos de lluvia, nuestro caso de este análisis. De igual modo los 50\% centrales, hubo amplitud de $440 \mathrm{~mm}$, dentro del período de análisis, lo que es significativo, mismo para un período de 30 años de datos.

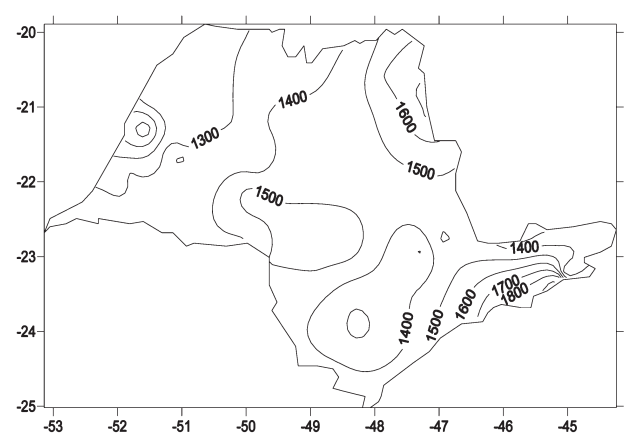

Figura 2. Isolíneas de valores medios, en el período estudiado.

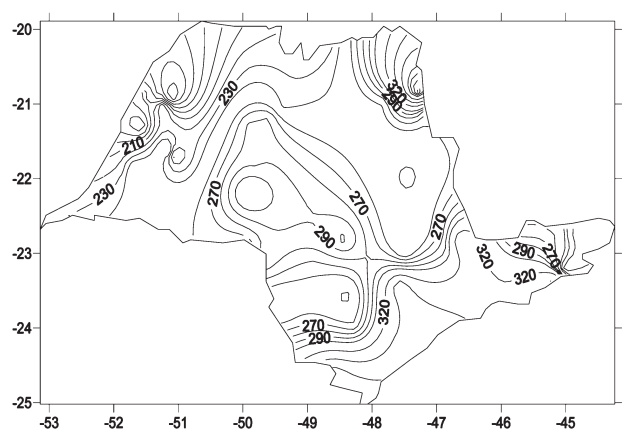

Figura 3. Isolíneas de los desviaciones estándar, en el período estudiado. 


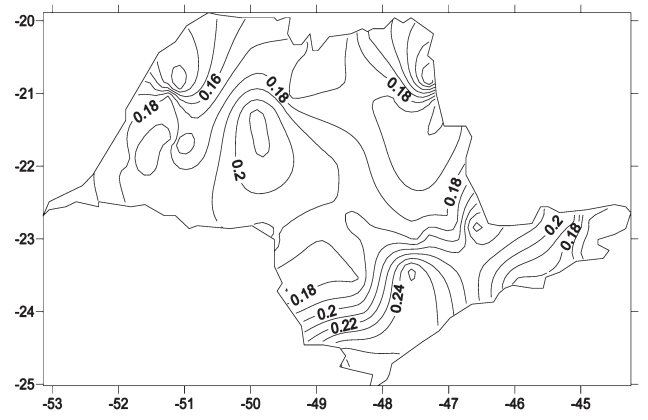

FIGURA 4. Isolíneas relativas al coeficiente de la variación, dentro del período analizado.

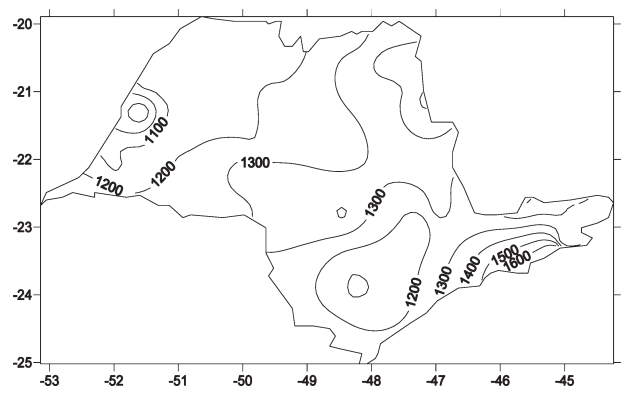

FIgURA 6. Isolíneas del cuartil inferior.

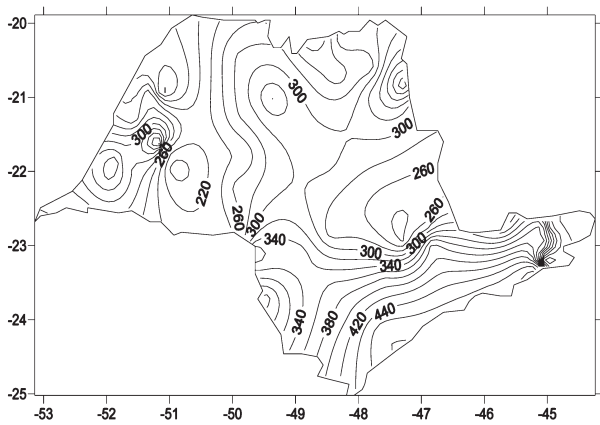

FIGURA 5. Isolíneas de las amplitudes intercuartil, dentro del período analizado.

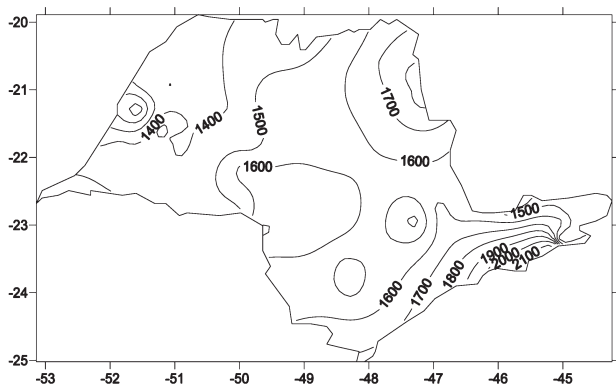

FIGURA 7. Isolíneas del cuartil superior.

En las Figuras 6 e 7 son trazadas las isolineas de los cuartiles inferior (Figura 6) e isolineas del cuartil superior (Figura 7). Considerando el análisis de los datos referentes al cuartil inferior (corresponde al 25\% de los datos analizados, o sea, 25\% corresponden a los datos más bajos de lluvias de la serie, se puede observar que los valores oscilaron entre $1.100 \mathrm{~mm}$ (al Oeste) y $1.600 \mathrm{~mm}$ (al Este). Esta es también una medida de variabilidad de una muestra analizada, en el caso de la precipitación pluvial. En la Figura 7, son trazadas isolineas referentes al cuartil superior, o sea, 25\% de muestra, en relación a los datos totales. En el caso de los cuartiles superiores se puede observar significativamente la variabilidad espacial com máximas en la costa (región Este del Estado) y mínima de 1.400 $\mathrm{mm}$, al Noroeste del referido Estado.

En la distribución espacial de las isolineas de máximas (Figura 8) y mínimas precipitaciones (Figura 9), se puede observar que la variabilidad entre esos valores esta bien marcada. Los valores máximos de lluvia, dentro del período analizada se concentran al Este, Sudeste y Sudoeste, con $2.400 \mathrm{~mm}$ de precipitación pluvial y al Oeste se pueden observar los menores valores, $1.600 \mathrm{~mm}$, aproximadamente, Figura 8. En la Figura 9, se puede observar variabilidades de valores mínimos de precipitación pluvial, con valores de $110 \mathrm{~mm}$, aproximadamente, al Sudeste del Estado y valores de $700 \mathrm{~mm}$, al Oeste del referido Estado.

Fueron trazadas isolineas de anomalías de precipitación para los años analizados en este trabajo, Figuras 10 a 20. Se puede observar significativa variabilidad de un año para 


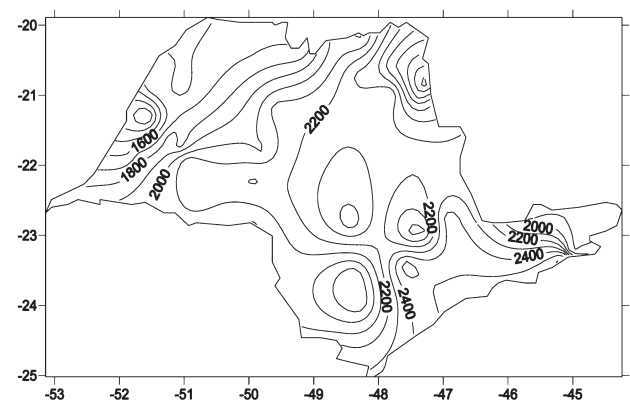

Figura 8. Isolíneas de valores máximos de precipitación pluvial.

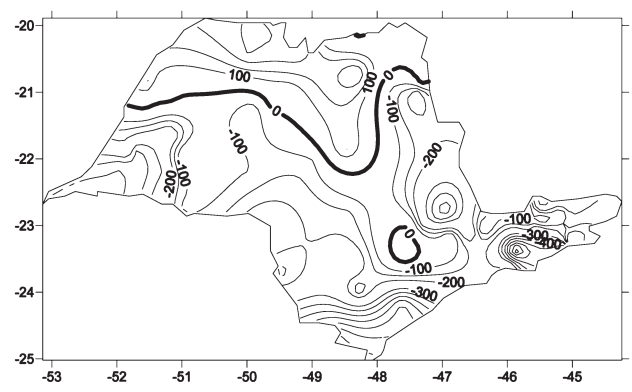

FIGURA 10. Isolíneas de anormalidades: 1978.

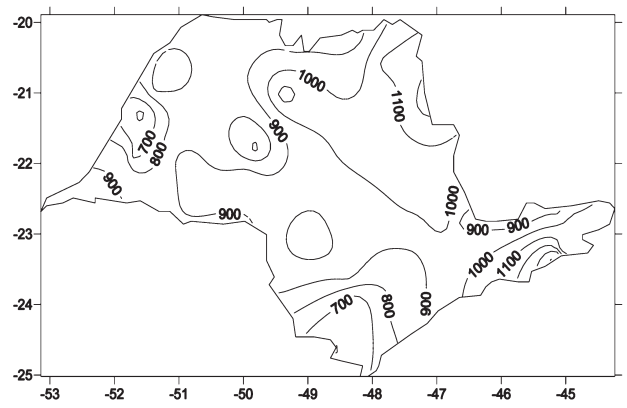

Figura 9. Isolíneas de valores mínimos de precipitación pluvial.

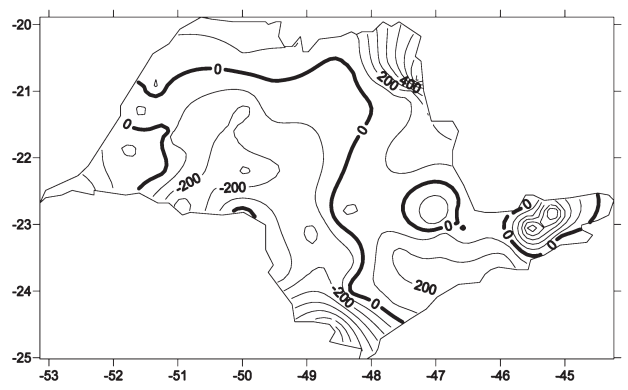

FIgURA 11. Isolíneas de anormalidades: año 1980.

otro, siendo que en algunos años, las lluvias fueron sobre la media climática y en otros bien abajo de esta media, o sea, en algunos años las lluvias fueron intensas, provocando excedente de agua en el Estado y en otros años, hubo escasees de lluvias.

Los años 1978 y 1980 fueron años con valores de lluvias inferiores a la media climática, pudiéndose observar que la región Norte y Noroeste presentaron valores de precipitaciones pluvial superiores a los valores medios del año 1978, no siendo, por lo tanto, valores muy superiores a las lluvias normales. Figura 10. También se puede observar lluvias bien inferiores a $300 \mathrm{~mm}$, al Este del Estado, para el año 1978. Eso muestra que mismo para un año específico, la variabilidad espacial de la precipitación pluvial es significativa. El año 1980 (Figura 11), presento valores de lluvias bien inferiores al valor medio climatológico, presentando una pequeña área, al Nordeste del Estado con precipitaciones sobre los 200 mm, comparativamente a la media climática.

Los años 1982 y 1983 fueron años de lluvias intensas, principalmente dentro del período de ocurrencia del evento El Niño - Oscilación Sur (ENOS) y se puede observar en las Figuras 12 y 13 que las isolineas de anormalidades para esos años fueron positivas en todo el estado, com valores superiores a $700 \mathrm{~mm}$ en el año 1983, al Este del referido estado. Otra observación importante es que la variabilidad espacial también fue significativa presentando en el mismo año, valores de $100 \mathrm{~mm}$ al Oeste del estado. Ya en el año 1982 (Figura 12) presento valores de anormalidades menores que 1983, pero también positivo en todo el estado. Al Este y al Sur del referido estado los valores da las isolineas de anormalidades fueron superiores a $500 \mathrm{~mm}$ (superiores a la media climatológica). 


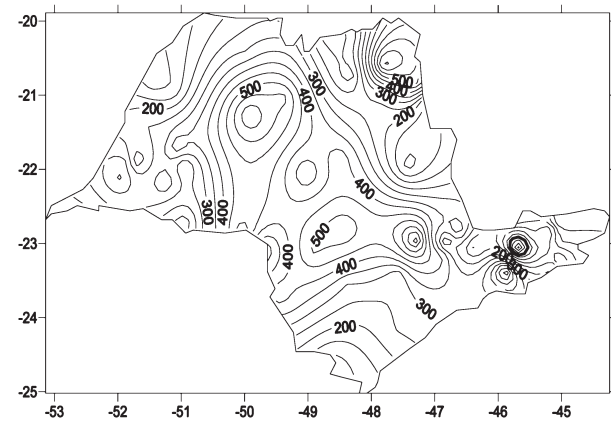

FIGURA 12. Isolíneas de anomalías: 1982.

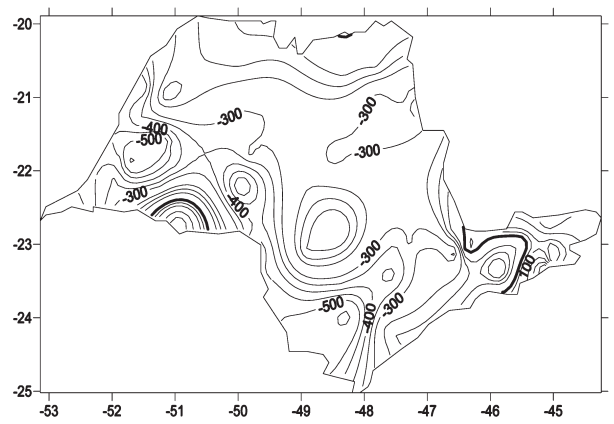

Figura 14. Isolíneas de anomalías: 1985.

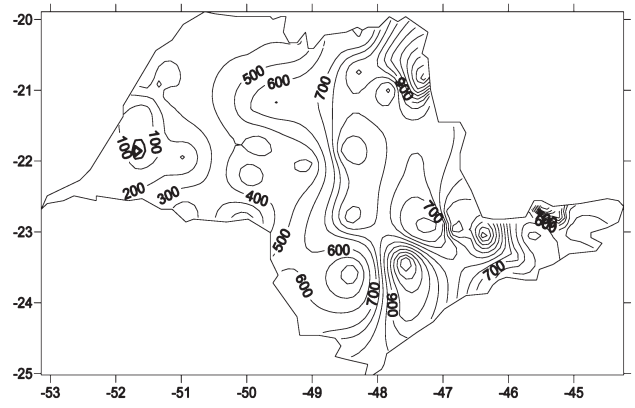

Figura 13. Isolíneas de anomalías: 1983.

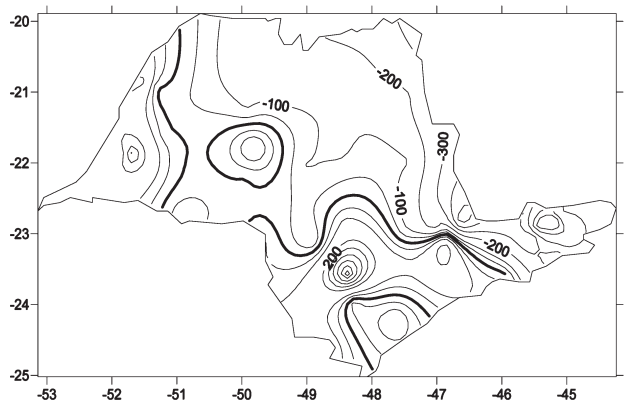

FIGURA 15. Isolíneas de anomalías: 1990.

En las Figuras 14 e 15, están presentadas las anormalidades de la precipitación pluvial para los años 1985 y 1990. El año 1985 presento anormalidades negativas y significativas en todo el estado, con valores inferiores a la media climática de $500 \mathrm{~mm}$ al Oeste Sudoeste del estado, Figura 14. El año 1985 fue un año con significativo déficit de lluvia en toda la región Sur y Sudeste del Estado. Ya el año 1990 presento significativa variabilidad espacial, con valores negativos en gran parte del estado, más con valores positivos al Sudoeste del mismo estado, Figura 15.

En las Figuras 16 y 17 son presentados anormalidades de precipitación pluvial para los años 1994 y 1997. Estos dos anos presentaron significativamente variabilidad de la lluvia, con valores inferiores a la media climática, aunque el ano 1997 (Figura 17) presente valores positivos, o sea, sobre la media climática, al Este y Noroeste del Estado. Ese año tiene un significado especial, pues se trata del ano del inicio de un intenso evento El Niño, aunque no tan pronunciado cuanto el evento de 1982 - 1983, para el Estado de São Paulo, de acuerdo a la comparación de las isolineas de las Figuras 12 e 13 con las Figuras 17 e 18.

En la figura 19 se presenta la anormalidad de la precipitación pluvial para el año 1998. Como se ha indicado, se trata de un año en que ocurrió un evento intenso El Niño, con inicio en 1997. Se puede observar que las isolíneas de anomalías de la precipitación pluvial no fueron positivas en todo el estado. Valores negativos de anormalidades están bien marcados en gran parte del Estado de São Paulo. De esta forma, se puede concluir que, ese evento haya sido considerado intenso, por los efectos provocados en diferentes regiones de 


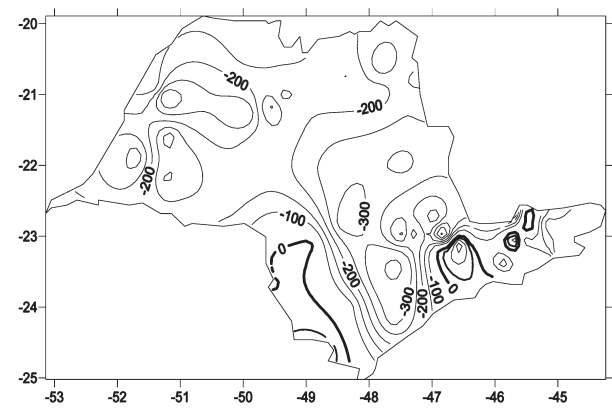

FIGURA 16. Isolíneas de anomalías: 1994.

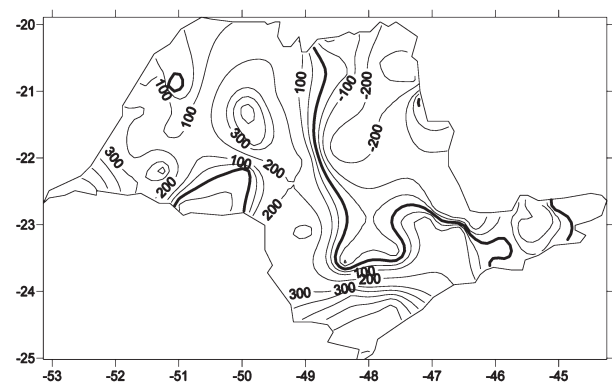

FIGURA 19. Isolíneas de anomalías: 1998.

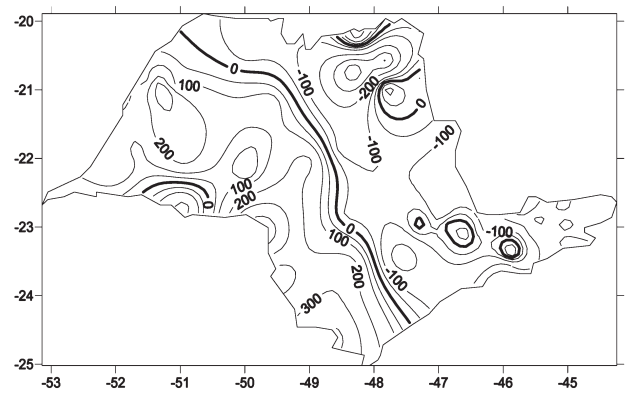

FIGURA 17. Isolíneas de anomalías: 1997.

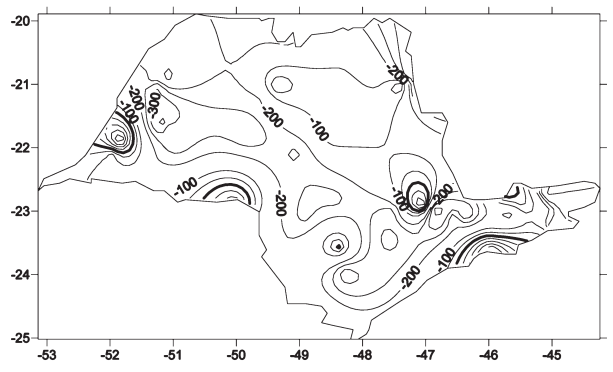

FIGURA 20. Isolíneas da anomalías: 2002.

la Tierra, en São Paulo, su actuación no fue tan marcada como el evento El Niño 1982/83, citado anteriormente. En la Figura 20, son presentadas anormalidades de la precipitación para el ano 2002, con valores negativos en todo el estado.

A través del análisis multivariado se determino el dendograma. Ese método tiene por finalidad clasificar áreas homogéneas. Fueron utilizados datos totales anuales de todas las series pluviométricas utilizadas en este trabajo y a través de un corte subjetivo en la Figura 21, se puede determinar seis áreas homogéneas en el estado. En la Figura 22, son presentadas esas áreas, pudiendo observar un área al Oeste (área de baja precipitación pluvial), un área mas al Este, con precipitaciones pluviales significativas, influenciada por sistemas dinámicos completamente diferentes de aquellos observados en la región Oeste (área tres), además de otras áreas con características continentales. Esta clasificación posibilita inferir que hay significativa variabilidad de la precipitación pluvial en todo el estado y cada una de las variabilidades espacial puede ser explicada por una dinámica climática asociada a diferentes características, tales como continentalísima, marítimas, entre otros.

En la Tabla 1 están presentados la estadística descriptiva para cada grupo. Esos cálculos fueron hechos con base en la media temporal de las estaciones pertenecientes a cada grupo. Por ejemplo: el grupo 1 fue clasificado con seis estaciones. Fueron calculados los valores medios, desviación estándar, coeficiente de variación y amplitud intercuartil para ese grupo, así como para los demás clasificados. Los valores de esas estadísticas presentan significativa variabilidad entre los grupos. Se puede observar que el grupo 1 presentó mayor 


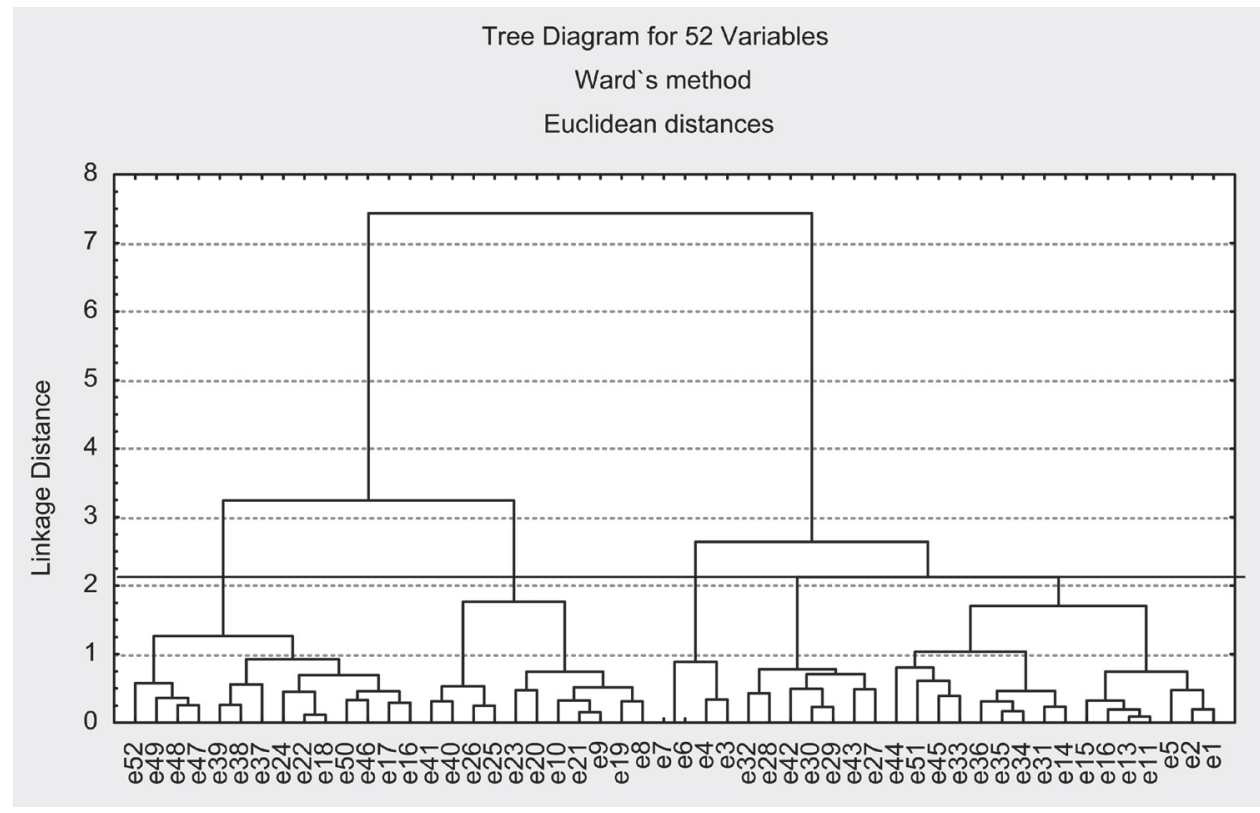

Figura 21. Dendograma generado a partir de la base de datos, totales anuales, dentro del período del estudio.

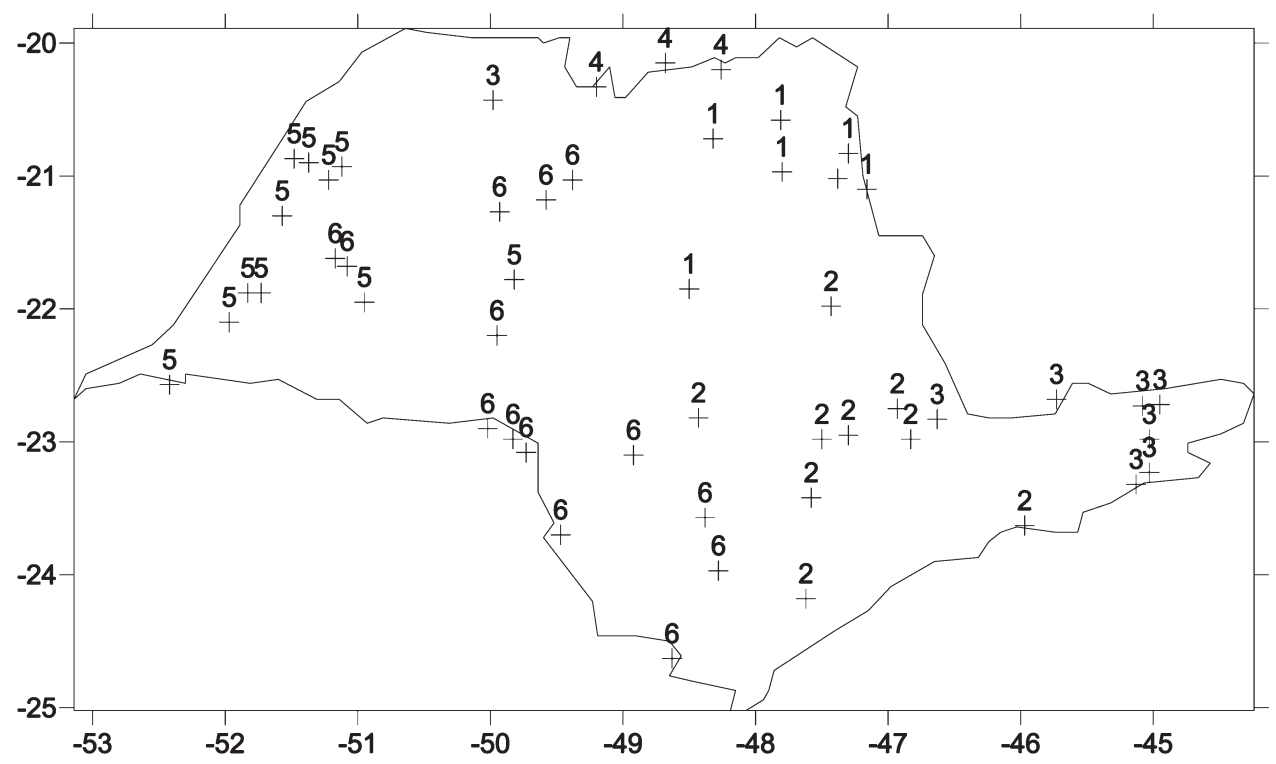

Figura 22. Clasificación de las áreas homogéneas entre las áreas de estudio. 
valor medio (150.9 mm), con mayor dispersión (260.2 mm). La explicación para esa mayor precipitación media está en el hecho de las series pluviométricas clasificadas en ese grupo estarán localizadas mas a la costa del Estado. Ya los grupos 4, 5 y 6, están localizados en áreas continentales, sufriendo la influencia de dinámicas climáticas distintas de aquellas relativas la grupo 1. Los coeficientes de variaciones de cada grupo no presentaron valores altos, mostrando que la variabilidad de estos grupos no esta marcada.

Se puede observar que hay variabilidad en la precipitación pluvial en todas las áreas elegidas, aunque esa variabilidad no sea marcada como puede ser observado en los valores de las amplitudes intercuartiles, en el desviación estándar y en los valores de los coeficientes de variación.

Tabla 1

ESTADÍSTICA DESCRIPTIVA PARA CADA GRUPO CLASIFICADO, SIENDO DP EL DESVÍO PADRÓN, CV EL COEFICIENTE DE VARIACIÓN Y AIQ LA AMPLITUD INTERCUARTIL DE LOS GRUPOS CLASIFICADOS

\begin{tabular}{|l|r|r|r|r|r|r|}
\hline & GRUPO 1 & GRUPO 2 & GRUPO 3 & GRUPO 4 & GRUPO 5 & GRUPO 6 \\
\hline Media & 1580.9 & 1474.8 & 1420.3 & 1370.8 & 1329.7 & 1406.3 \\
\hline DP & 260.2 & 236.8 & 200.7 & 212.6 & 155.0 & 208.9 \\
\hline CV (\%) & 16.0 & 16.0 & 14.0 & 16.0 & 12.0 & 15.0 \\
\hline AIQ & 278.7 & 316.3 & 258.3 & 253.1 & 237.3 & 264.4 \\
\hline
\end{tabular}

En la Tabla 2 están presentados algunos valores temporales y espaciales, para cada área clasificada. Esos valores representan las medias de las series pluviométricas de todas las estaciones pertenecientes a cada grupo. Los años escogidos forman aquellos que presentaron alguna particularidad, tales como el evento El Niño (1982/83), un año seco (1985) y un año con gran variabilidad en la precipitación pluvial del Estado (1990)

Tabla 2

VALORES MEDIOS PARA GRUPO Y PARA ALGUNOS AÑOS ESCOGIDOS CON BASE EN LA VARIABILIDAD DE LAS ANOMALÍAS

\begin{tabular}{|l|l|l|l|l|l|l|}
\hline $\mathbf{1 9 8 2}$ & 1944.6 & 1839.9 & 1669.5 & 1570.5 & 1511.5 & 1807.3 \\
\hline $\mathbf{1 9 8 3}$ & 2481.2 & 2289.6 & 1969.3 & 1906.6 & 1500.1 & 1889.6 \\
\hline $\mathbf{1 9 8 5}$ & 1330.0 & 1243.0 & 1591.2 & 1331.0 & 1003.6 & 1001.4 \\
\hline $\mathbf{1 9 9 0}$ & 1384.0 & 1426.9 & 1163.1 & 1168.5 & 1439.4 & 1424.2 \\
\hline
\end{tabular}

Con base en el dendograma de las matrices temporales de las series totales pluviales, fueron agrupados todos los años, dentro del período de estudio, resultando en los siguientes agrupamientos: Grupo 1 (años: 1976, 1982 y 1983); Grupo 2 (años: 1975, 1978, 1981, 1984 , 1985, 1994, 1999, 2002 y 2003); Grupo 3 (años: 1979, 1980, 1986, 1988, 1991, 1995, 1996 y 2000) y Grupo 4 (años: 1974, 1977, 1987, 1989, 1990, 1992, 1993, 1997, 1998 у 2001). 
Esos agrupamientos muestran que hay variabilidades temporales significativas dentro de las áreas analizadas, generando de esta forma, cuatro grupos bien distintos dentro del Estado.

Las Figuras 23 a 28 analizan la evolución temporal (anual) a través del índice de precipitación pluvial representativo de cada área homogénea. Se puede observar significativa variabilidad de la lluvia de año para año y espacialmente, con los grupos 5 y 6 (al Oeste del Estado), presentando menores valores del índice, o sea, menores lluvias en las dos áreas. Los

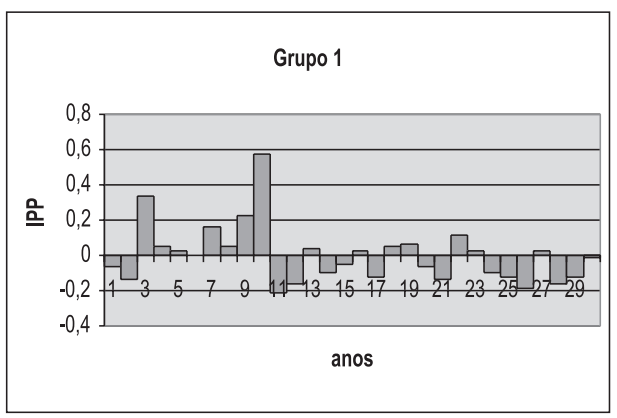

Figura 23. Análisis de la precipitación pluvial para el área homogénea (Grupo 1), generada con base en el análisis multivariado (cluster).

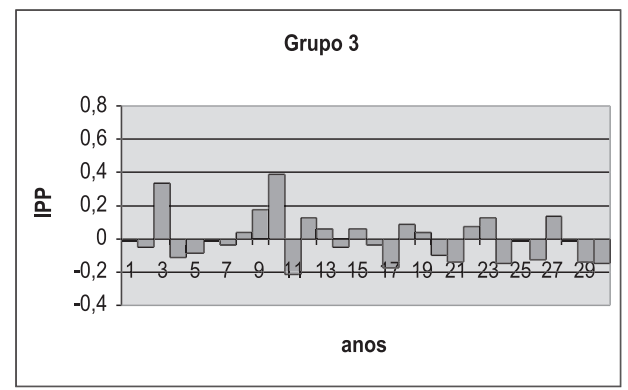

Figura 25. Análisis de la precipitación pluvial para el área homogénea (Grupo 3), generada con base en el análisis multivariado (cluster).

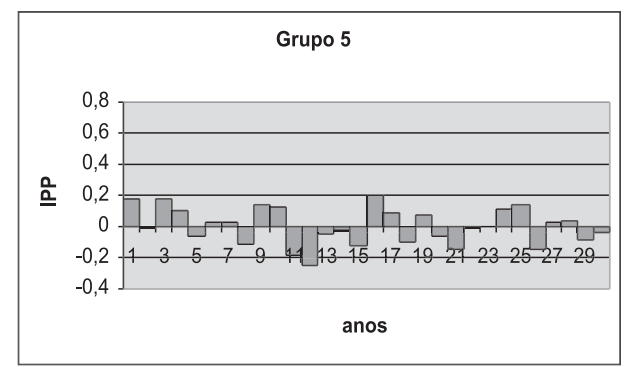

FIGURA 27. Análisis de la precipitación pluvial para el área homogénea (Grupo 5), generada con base en el análisis multivariado (cluster).

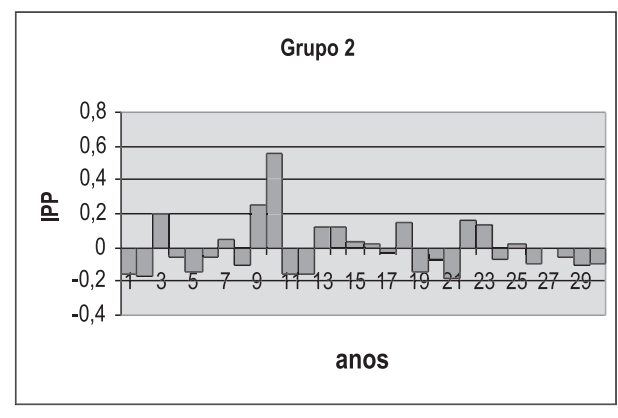

Figura 24. Análisis de la precipitación pluvial para el área homogénea (Grupo 2), generada con base en el análisis multivariado (cluster).

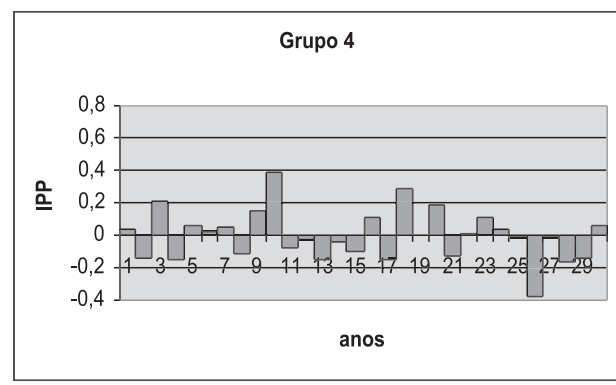

Figura 26. Análisis de la precipitación pluvial para el área homogénea (Grupo 4), generada con base en el análisis multivariado (cluster).

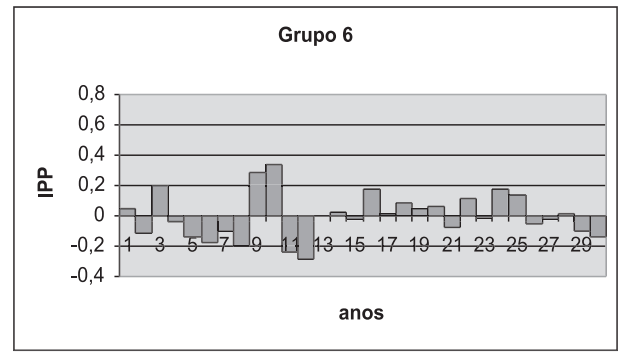

Figura 28. Análisis de la precipitación pluvial para el área homogénea (Grupo 6), generada con base en el análisis multivariado (cluster). 
años analizados fueron desde 1974 a 2003, pudiéndose observar que el año 1983 presenta mayor índice en los grupos 1 y 2, no presentan señales significativas en las demás áreas homogéneas. Los grupos 3 y 4 presentan algunas señales para el año 1983, demostrando una transición entre la costa y la parte mas continental del Estado.

\section{Conclusión}

El Estado de São Paulo presenta significativa variabilidad espacial y temporal de la precipitación pluvial.

Gran parte de esa variabilidad temporal puede ser explicada por la ocurrencia de los eventos El Niño y La Niña que afectan las lluvias de ese Estado.

El Estado de São Paulo presentó 6 áreas homogéneas, con el grupo 3 y 5 presentando marcada variabilidad.

\section{Referencias bibliográficas}

ALVES, M. L., MARENGO, A. J., JUNIOR, C. H. CASTRO, C. Início da estação chuvosa na região sudeste do Brasil: Parte 1 - Estudos observacionais. Revista Brasileira de Meteorologia, São Paulo, v. 20, n. 3, p. 385-394, dez. 2005.

ARAÚJO, M. A. A.; SANTOS, A.P. dos; VIOLA, P. E. Aspectos sinóticos das chuvas intensas sobre a região sudeste o Brasil, fevereiro de 1988. CONGRESSO BRASILEIRO DE METEOROLOGIA, 5, Anais... Rio de Janeiro 7 a 11 de novembro de 1988, v. 2, pp. 39-43. 1988.

ASSUNÇÃO, W.L. Análise climática da duração e extensão da grande seca de 1963 no oeste mineiro. In: VI Simpósio Brasileiro de Climatologia Geográfica, 6., 2004, Aracaju. Anais... Aracaju: UFS, 2004. 1- CD-ROM.

CAVALCANTE, I. F. A.; FERREIRA, N. J.; KOUSKY, V. E. Análise de um caso de atividade convectiva associado a linhas de instabilidade na região Sul e Sudeste do Brasil. INPE - 2574 - PRE /222. 1982.

COULSON, K. L. Solar and terrestrial Radiation. Academic San Francisco, 1975.

FERREIRA, C.C.M.; BATISTA, A.J. Distribuição espaço-temporal de veranicos e deficiência hídrica na bacia do rio Paraibuna - MG: variabilidade, probabilidade e processos intervenientes. In: VI Simpósio Brasileiro de Climatologia Geográfica, 6., 2004, Aracaju. Anais... Aracaju: UFS, 2004. 1- CD-ROM.

MINUZZI, R. B., SEDIYAMA, G. C., COSTA, J. M. N., RIBEIRO, A. Influência do fenômeno La Niña nos veranicos do Estado de Minas Gerais. Revista Brasileira de Agrometeorologia, Santa Maria, v. 12, n. 2, p. 341-348. 2004.

MINUZZI, R.B.; SEDIYAMA, G.C.; COSTA, J.M.N.; RIBEIRO, A. Anomalias de precipitação durante o período chuvoso no Estado de Minas Gerais em eventos El Niño e La Niña. In: XIII Congresso Brasileiro de Agrometeorologia, 13, 2003, Santa Maria, RS. Anais... Santa Maria, RS: UFRS, 2003. 1 - CD-ROM.

MONTEIRO, C. A. de F. A dinâmica climática e as chuvas no Estado de São Paulo: estudo geográfico sob a forma de Atlas. São Paulo: IG/USP, 1973.

NERY, J. T.; SILVA, E. S.; CARFAN, A. C. Distribuição da precipitação pluvial no Estado de São Paulo. In: VI Simpósio Brasileiro de Climatologia Geográfica, 6, 2004, Aracaju. Anais... Aracaju: UFS, 2004. 1- CD-ROM.

NOBRE, C. A. Ainda sobre a Zona de Convergência do Atlântico Sul. A importância do Oceano Pacífico. Climanálise, 1988.

QUADRO, M. F. L. Estudo de episódios de Zonas de Convergência do Atlântico Sul (ZCAS) sobre a América do Sul. Dissertação de Mestrado, INPE, São José dos Campos (SP), 1994.

REIS, E. Estatística multivariada aplicada. Lisboa: Silabo, 1997.

RICHMAN, M.B. Rotation of principal components: a reply. Londres: Journal of Climatology, 1986. 